\title{
De novo assembly and Characterisation of the Transcriptome during seed development, and generation of genic-SSR markers in Peanut (Arachis hypogaea L.)
}

\author{
Jianan Zhang ${ }^{1,2}$, Shan Liang ${ }^{3}$, Jialei Duan ${ }^{4}$, Jin Wang ${ }^{1}$, Silong Chen ${ }^{1}$, Zengshu Cheng ${ }^{1}$, Qiang Zhang ${ }^{1}$, \\ Xuanqiang Liang ${ }^{5}$ and Yurong $\mathrm{Li}^{\mathrm{i}^{*}}$
}

\begin{abstract}
Background: The peanut (Arachis hypogaea L.) is an important oilseed crop in tropical and subtropical regions of the world. However, little about the molecular biology of the peanut is currently known. Recently, next-generation sequencing technology, termed RNA-seq, has provided a powerful approach for analysing the transcriptome, and for shedding light on the molecular biology of peanut.

Results: In this study, we employed RNA-seq to analyse the transcriptomes of the immature seeds of three different peanut varieties with different oil contents. A total of 26.1-27.2 million paired-end reads with lengths of $100 \mathrm{bp}$ were generated from the three varieties and 59,077 unigenes were assembled with N50 of $823 \mathrm{bp}$. Based on sequence similarity search with known proteins, a total of 40,100 genes were identified. Among these unigenes, only 8,252 unigenes were annotated with 42 gene ontology $(\mathrm{GO})$ functional categories. And 18,028 unigenes mapped to 125 pathways by searching against the Kyoto Encyclopedia of Genes and Genomes pathway database (KEGG). In addition, 3,919 microsatellite markers were developed in the unigene library, and 160 PCR primers of SSR loci were used for validation of the amplification and the polymorphism.

Conclusion: We completed a successful global analysis of the peanut transcriptome using RNA-seq, a large number of unigenes were assembled, and almost four thousand SSR primers were developed. These data will facilitate gene discovery and functional genomic studies of the peanut plant. In addition, this study provides insight into the complex transcriptome of the peanut and established a biotechnological platform for future research.
\end{abstract}

\section{Background}

The peanut (Arachis hypogaea L.), also known as the groundnut, is an important oilseed crop in the tropical and subtropical regions of the world. It is grown on six continents but mainly in Asia, Africa and America. Peanuts are cultivated on 23.51 million hectares worldwide, with a total global production of approximately 35.52 million tons (the weight includes the shell). China is the largest producer in the world, accounting for $37.6 \%$

\footnotetext{
* Correspondence: liyrl@163.com

'Institute of Food and Oil Crops, Hebei Academy of Agriculture and Forestry Sciences/Laboratory of Crop Genetics and Breeding of Hebei Province,

Shijiazhuang 050031, China

Full list of author information is available at the end of the article
}

(13.34 million tons) of the total world production (FAO, 2009, http://faostat.fao.org).

Peanuts have a desirable fatty acid profile and are rich in vitamins, minerals and bioactive materials, including several known heart-healthy nutrients, such as monounsaturated and polyunsaturated fatty acids, potassium, magnesium, copper niacin, arginine, fibre, $\alpha$-tocopherol, folates, phytosterols, and flavonoids. Indeed, peanut consumption has been associated with an improvement in the overall quality of the diet and nutrient [1-4].

In China, almost $60 \%$ of the peanuts are used to produce peanut oil [5]. Peanut oil, due to its high monounsaturated fat content, is considered healthier than saturated oils and is resistant to rancidity. Monounsaturated fat, 
much of which is oleic acid, is a healthy type of fat that has been implicated in the health of skin [6] and has been demonstrated to reduce cardiovascular disease risk and/or risk factors in both epidemiological and clinical studies $[1,2,7]$.

The development of the peanut seed has been studied intensely to understand the physiological, biochemical, and molecular characteristics that determine the oil quality and their beneficial nutritional contributions. However, the development of the peanut seed is a complex process involving a cascade of biochemical changes, which involve the transcriptional modulation of many genes, yet little is known about these transcriptional changes and their regulation. To date, little research in this area has been reported. Bi [8] developed a seed cDNA library for the peanut to analyse gene expression levels during seed development, and 17,000 expressed sequence tags (ESTs) were sequenced and used for microarray analysis. Recently, the development of nextgeneration high-throughput DNA sequencing technology has provided a novel method for both mapping and quantifying transcriptomes (RNA-seq) [9]. RNA-seq technology has been successfully applied quite ubiquitously to species such as humans, yeast, mice, grape, Arabidopsis, rice, soybeans, sesame, and sweetpotato [9-19]. Moreover, RNA-seq data are highly reproducible, with few systematic discrepancies among technical replicates [20]. The latest paired-end tag sequencing strategy of RNA-seq further improves the DNA sequencing efficiency and expands short-read lengths, providing a better depiction of transcriptomes [21]. Transcriptomic information is used in a wide range of biological studies and provides fundamental insight into biological processes and applications, such as the levels of gene expression [22], the gene expression profiles during development $[13,17]$ or after experimental treatments [23], gene discovery [24], SSR mining [10,11,25], and SNP discovery [12,25-27]. However, transcriptomic information is lacking for the peanut plant because this information is difficult to obtain and, to date, there has been little interest in such data.

Chen [28] reported that the accumulation of seed oil in peanuts could be divided into three stages based on phenotype, namely, the initial accumulation stage, the fast accumulation stage and the steady accumulation stage. As we are interested in identifying genes that are expressed in the seed during the fast accumulation period, we carried out a global analysis of the peanut transcriptome during seed development using the Illumina RNA-seq method. We also present an overview of the RNA-seq data for the peanut as a potential model for future RNA-seq analyses and to establish a biotechnological platform for peanut research.

\section{Methods}

\section{Sample Preparation and Sequencing}

Three peanut varieties, which greatly differ in oil content but are similar in mature process, were used in this study. Jihua 4 (JH4), a variety with a high oil content (57\%), as determined in a 2009 field experiment in Hebei, China, was bred at the Hebei Institute of Agricultural Sciences and is widely grown in Northern China. Kaixuan01-6 (K01), a germplasm resource with a similar oil content (52\%), also determined in the above field experiment in Hebei, was provided by the Yantai Institute of Agricultural Sciences in the Shandong Province of China. Te21 (T21), a germplasm resource with a low oil content (48\%), as determined in China, was also used.

For each variety, RNA was isolated from ten immature seeds of five plants, which were harvested at 7 weeks after flowering during the 2010 growing season from a farm in Hebei province (Shijiazhuang, Hebei, China). Total RNA was extracted using a previously described method [20]. The RNA quality and quantity were determined using an Agilent 2100 Bioanalyzer (Agilent Technologies, Santa Clara, CA). Beads coated with oligo(dT) were used to isolate poly (A) mRNA after the total RNA was collected. Fragmentation buffer (Ambion, Austin, TX) was added to digest the mRNA to produce short fragments. The first strand of cDNA was synthesised using random hexamer primers, followed by synthesis of the second strand. The short fragments were purified with the QIAquick PCR Purification kit (Qiagen, Valencia, CA) for both end repair and the poly (A) addition reaction. The purified DNA libraries were amplified by PCR for 18 cycles. Finally, Solexa HiSeq ${ }^{\mathrm{TM}} 2000$ was employed to sequence the libraries using PCR amplification (BGI, Shenzhen, China).

\section{De novo Assembly and Analysis of Illumina Reads}

The samples were assembled with SOAPdenovo [29] separately. The numbers of paired-end Illumina reads of JH4, K01, and T21 were 27,159,362, 26,938,530, and $26,142,148$, respectively. The reads were first combined to form longer fragments, i.e., contigs. The reads were then mapped back to the contigs, and the paired-end reads and contigs from the same transcript were assembled to form a longer sequence, with $\mathrm{N}$ for unknown sequences (i.e., scaffolds). Paired-end reads were again used for gap filling of the scaffolds to obtain unigenes with the least Ns that could not be extended on either end. For future analyses, the unigenes from the three samples were assembled again to acquire nonredundant unigenes (All-Unigenes) that were as long as possible. The All-Unigenes assembled from the three samples were compared with the NCBI non-redundant 
(NR) protein database using blastx v2.2.14 [30] with an E-value cut-off of $1 \mathrm{e}^{-5}$. Based on the results of the protein database annotation, Blast2GO [31] was employed to obtain the functional classification of the unigenes based on GO terms. WEGO software [32] was used to perform the GO functional classification for all of the unigenes and to understand the distribution of the gene functions of this species at the macro level. The KEGG database (V56.0, Oct. 1, 2010) [33] was used to annotate the pathway of these unigenes.

\section{SSR mining and primer design}

We employed MIcroSAtellite (MISA) http://pgrc.ipk-gatersleben.de/misa/ for microsatellite mining. In this study, the SSRs were considered to contain motifs with two to six nucleotides in size and a minimum of 5 contiguous repeat units. Based on MISA results, Primer3 v2.23 (http://primer3.sourceforge.net) was used to design the primer pairs with default setting, and the PCR product size ranging from 100 to $280 \mathrm{bp}$. Six varieties were selected to validated the polymorphism of 160 random SSR markers. The six varieties included three varieties (Jihua 2, Jihua 5, and SW9721), which were all bred at the Hebei Institute of Agricultural Sciences, and the three varieties (JH4, K01, T21) used in this study. The SSR data on the six varieties were obtained following the methods described by Liang [34].

\section{Results and Discussion}

Sequencing and De novo Assembly of Solexa Short Reads We generated 27.2, 26.9 and 26.1 million 100-bp pairedend reads for the JH4, $\mathrm{K} 01$ and T21 varieties, encompassing 2.44, 2.42 and $2.35 \mathrm{~Gb}$ of sequence data, respectively (Table 1). The GC contents of the three varieties were $48.94 \%, 49.05 \%$, and $47.94 \%$, respectively.

Assembling these reads produced 44,028, 47,110 and 44,157 unigenes for the $\mathrm{JH} 4, \mathrm{~K} 01$ and T21 varieties, respectively; only unigenes greater than $200 \mathrm{bp}$ in length were further analysed. The N50 values of these three unigenes were $664 \mathrm{bp}, 616 \mathrm{bp}$ and $616 \mathrm{bp}$, respectively. After the final clustering, 59,077 unigenes were obtained, with approximately equal contribution from varieties $\mathrm{JH} 4$ (74.53\%), K01 (79.74\%), and T21 (74.74\%) (The peanut transcriptome sequences is available at National Centre for Biotechnology Information (NCBI) Transcriptome Shotgun Assembly (TSA) database, http://www.ncbi.nlm. nih.gov/, accession numbers are JR540742-JR590649). The length of the unigenes varied from $200 \mathrm{bp}$ to $10654 \mathrm{bp}$, with an average of $619 \mathrm{bp}$, and the N50 value was $823 \mathrm{bp}$. The majority of the reads were in the range of 201-500 bp ( $64.80 \%$ of the unigenes), and 46,176 unigenes $(78.16 \%$ of all of the unigenes) were shorter than $1 \mathrm{k}$. These results should provide a sequence basis for future studies, such as gene cloning and transgenic studies.

\section{Characterisation of the unigenes}

Among the 59,077 unigenes, the sequence directions of 42,997 of the unigenes were determined using blastx against the NCBI non-redundant (NR), Swiss-Prot, KEGG, and clusters of orthologous groups (COG) of protein databases with an E-value cut-off of $1 \mathrm{e}^{-5}$. In addition, the protein coding regions of 39,204 unigenes were predicted.

The unigenes were compared against the NCBI NR protein database using blastx. Among the 59,077 unigenes, $40,100(67.88 \%)$ had at least one significant match with an E-value below $1 \mathrm{e}^{-5}$. A total of 18,977 unigenes had no significant matches to any known protein, the result that may be partly due to novel genes or highly divergent genes, or these unigenes could represent untranslated regions. For validating redundancy of the data set with publicly available data, sequence similarity search was conducted against the NCBI Unigene database $\mathrm{ftp}: / / \mathrm{ftp} . \mathrm{ncbi}$. nlm.nih.gov/repository/UniGene/Arachis_hypogea/ using blastn with an E-value cut-off of $1 \mathrm{e}^{-10}$. The results indicated that out of 59,077 unigenes, 34,815 (58.93\%) showed significant similarity to publicly unigenes database. All the information on the redundancy of the data set with the publicly available data were showed in the supplemental file (Additional file 1: Table S1).

We identified novel transcribed sequences using blastn and the NCBI mRNA database sequences for peanut with an E-value cut-off of $1 \mathrm{e}^{-10}$. A total of 24,814 $(42.00 \%)$ of the unigenes did not significantly match the mRNA database and were, thus, considered putative novel transcribed sequences (Figure 1). The lengths of the novel unigenes varied from $200 \mathrm{bp}$ to $6267 \mathrm{bp}$, with an N50 value of $288 \mathrm{bp}$. Among these novel unigenes, only $1,046(4.2 \%)$ were longer than $1 \mathrm{~kb}$. A total of 14,407 (58.1\% of the novel transcribed sequences) of the unigenes had at least one significant match against the NCBI NR protein database. RT-PCR was carried out to validate these expression distributions further by selecting 10 random novel unigenes (Figure 2). The result indicated that all ten unigenes got right amplifications. The

Table 1 Summary of the short reads and the assemblies for three varieties.

\begin{tabular}{cccccc}
\hline Variety & Total Reads & Total Nucleotides (nt) & GC Percentage & Unigenes & Contribute to All-Unigenes \\
\hline JH4 & $27,159,362$ & $2,444,342,580$ & $48.94 \%$ & 44,028 & $74.53 \%$ \\
K01 & $26,938,530$ & $2,424,467,700$ & $49.06 \%$ & 47,110 & $79.74 \%$ \\
T21 & $26,142,148$ & $2,352,793,320$ & $47.94 \%$ & 44,157 & $74.74 \%$ \\
\hline
\end{tabular}




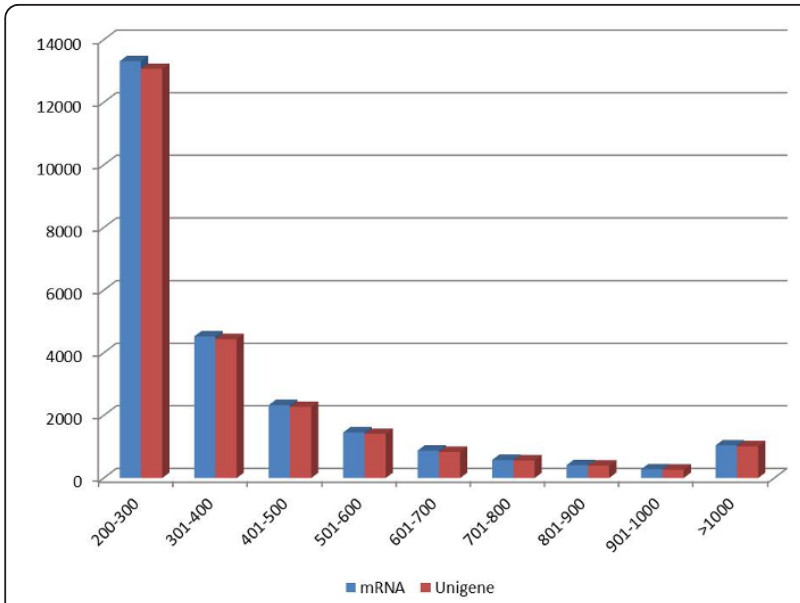

Figure 1 Characterization of nonredundancy of the unigenes set with publicly available data. mRNA means the

nonredundancy of unigenes set with NCBI peanut mRNA database; Unigene means the nonredundancy of unigenes set with NCBI peanut unigene database.

new information provided by this study could be useful to further peanut research.

\section{Functional classification of the peanut unigenes}

Of the 40,100 annotated unigenes, only 8,252 of the unigenes could be assigned at least one GO term, indicating that the peanut plant differs from model plants on genetic basis, and the search methodology of GO analysis is not suitable for peanut. These 8,252 unigenes were grouped into $42 \mathrm{GO}$ functional categories (http://www. geneontology.org), which are distributed under the three main categories of Molecular Function $(9,207)$, Biological Process $(5,660)$ and Cellular Components $(6,739)$ (Figure 3). Within the Molecular Function category,

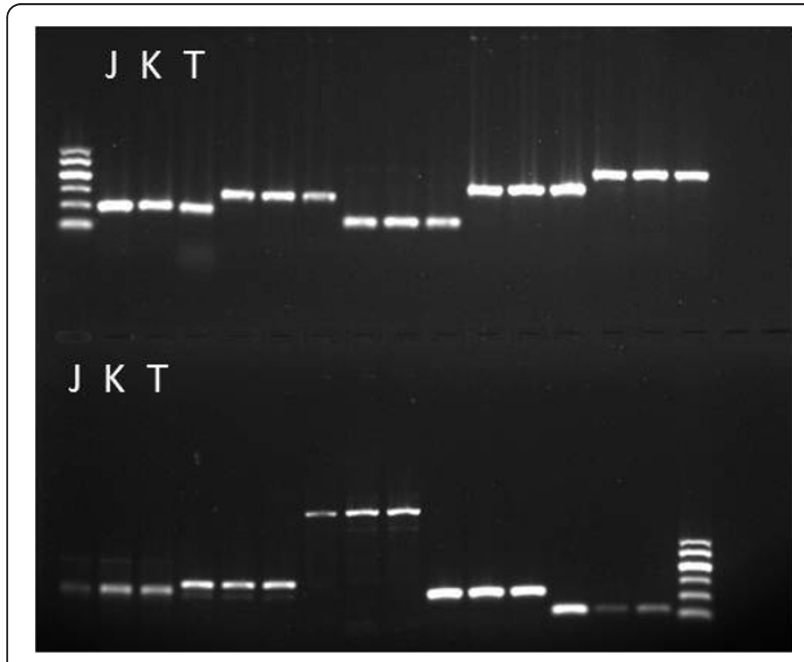

Figure 2 Validation of novel transcribed sequences. genes encoding binding proteins $(43.45 \%)$ and proteins related to catalytic activity (39.88\%) were the most enriched. Proteins related to metabolic processes $(30.42 \%)$ and cellular processes (29.6\%) were enriched in the Biological Process category. With regard to the Cellular Components category, the cell $(33.02 \%)$ and cell part $(33.01 \%)$ were the most highly represented categories. A total of 146 genes were annotated with the category of lipid biosynthetic process (GO: 0008610), and 69 genes were classified into the fatty acid biosynthetic process group (GO: 0006633) in the next level. Further analyses of these genes should provide information about fatty acid metabolism in peanuts.

A total of 18,028 unigenes were annotated with 125 pathways in the KEGG database (V56.0, Oct. 1, 2010); metabolic pathways were the most enriched $(3,899)$, followed by plant-pathogen interaction pathways $(1,290)$. Some pathways, such as the fatty acid metabolism pathway and fatty acid biosynthesis, the functions of which are clearly linked to the changes in the seed oil that take place during peanut ripening, would characterise in more detail in another paper (Zhang et al., unpublished).

\section{SSR mining from the peanut seed transcriptome}

Microsatellite markers (SSR markers) are some of the most successful molecular markers in the construction of a peanut genetic map and in diversity analysis. In this study, 5,883 microsatellites were detected in 4,993 unigenes, of which, 728 sequences contained more than 1 SSR. The microsatellites included 2,120 (36.0\%) dinucleotide motifs, 3,506 (59.6\%) trinucleotide motifs, 166 (2.8\%) tetranucleotide motifs, $42(0.7 \%)$ pentanucleotide motifs and $49(0.8 \%)$ hexanucleotide motifs (Figure $4 \mathrm{~A})$. The most abundant repeat type was (AG/CT), followed by (AAG/CTT), (ATC/ATG), (ACC/GGT), (AAC/GTT) and (AGG/CCT), respectively. (Figure 4B). Based on the 5,883 SSRs, 3,919 primer pairs were successfully designed using Primer3 (Additional file 2: Table S2). A total of 160 primer pairs (Additional file 3: Table S3) were randomly selected to validate these polymorphisms in six varieties. All 160 of the markers yielded amplification products, and 65 (40.63\%) exhibited polymorphisms among the six varieties.

\section{Conclusion}

In this study, we performed a global characterisation of the peanut transcriptome by RNA-seq using next-generation Illumina sequencing. We generated 26.1-27.2 million paired-end reads, comprising 59,077 unigenes from three different varieties of peanut with different oil contents. These unigenes were annotated with $42 \mathrm{GO}$ functional categories and 125 pathways. A total of 5,883 microsatellites were identified among the 59,077 unigenes, and 3,919 primer pairs were developed based on the sequence library. These data will facilitate gene discovery and 


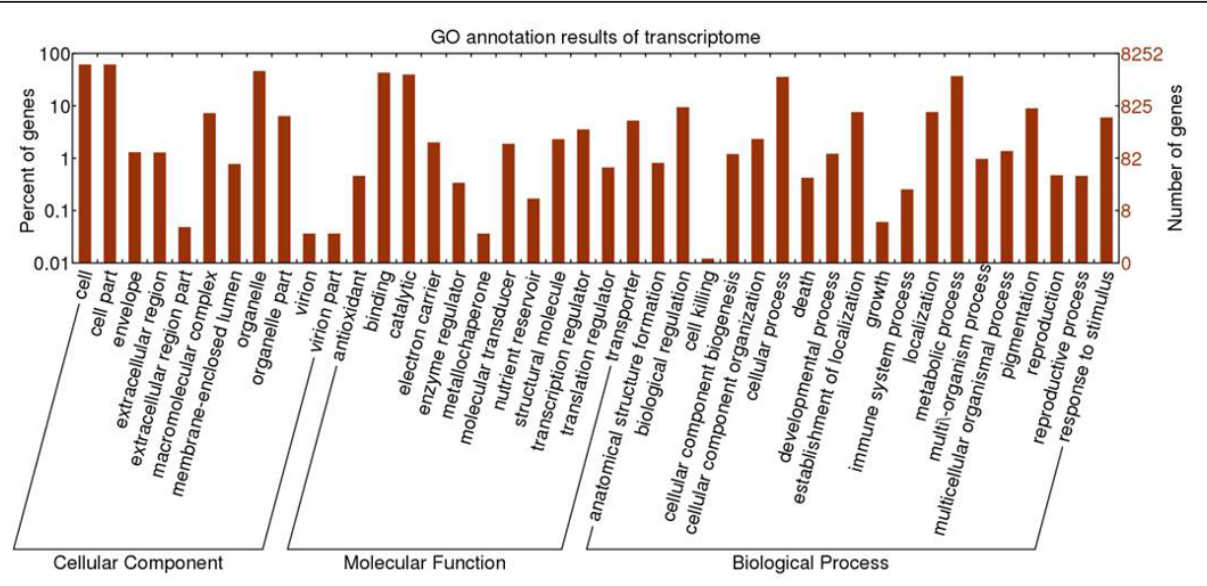

Fig. $3 \mathrm{GO}$ annotation results of transcriptome

Figure 3 Go annotation results of transcriptome.

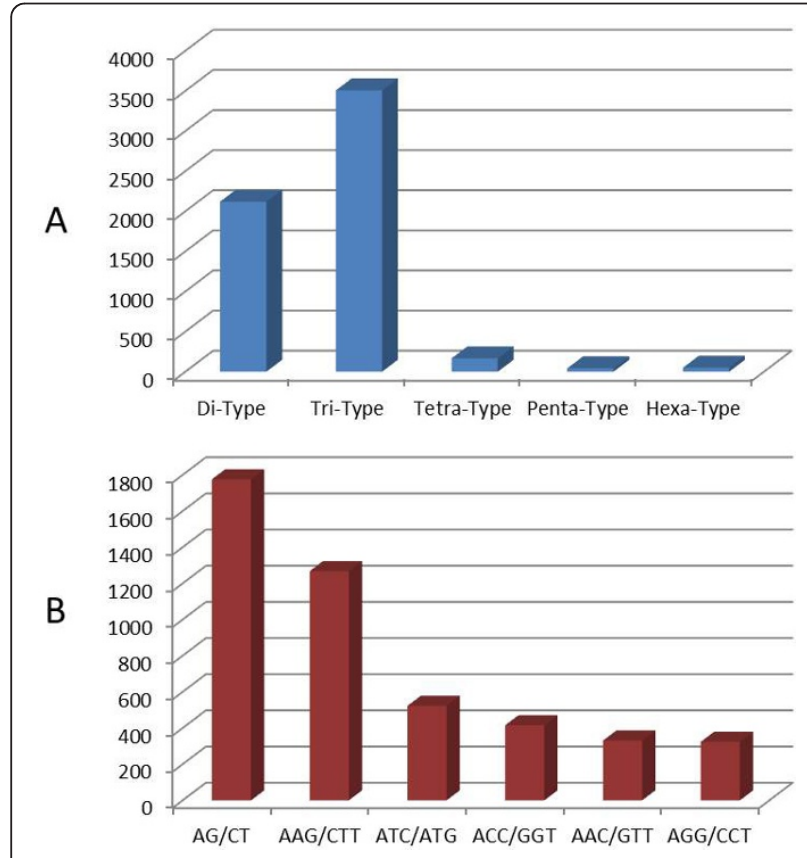

Figure 4 Characterization of SSRs mining. (A) Distribution to different repeat type classes; (B) Frequency of classified repeat types.

functional genomic studies in peanuts. We gained insight into the complex transcriptome of the peanut and established a biotechnological platform for future research.

\section{Additional material}

Additional file 1: Table S1. The information on the redundancy of the data in this study with publicly available data.

Additional file 2: Table S2. The primer pairs were successfully designed by Primer3 for all microsatellite.
Additional file 3: Table S3. 160 random markers were used to validate the amplification and polymorphism.

\section{Acknowledgements}

This work was supported by the earmarked fund for China Agriculture Research System (CARS-14), the Research Program on Quality-Improvement and Profit- Incensement for Major Economic Crops in Hebei Province (2011055005) and the Key Basic Research Program of Hebei Province (10960122D).

\section{Author details}

'Institute of Food and Oil Crops, Hebei Academy of Agriculture and Forestry Sciences/Laboratory of Crop Genetics and Breeding of Hebei Province, Shijiazhuang 050031, China. ${ }^{2}$ National Millet Improvement Center of China, Institute of Millet Crops, Hebei Academy of Agriculture and Forestry Sciences, Shijiazhuang 050031, China. ${ }^{3}$ Protein Science Laboratory of the Ministry of Education, School of Life Sciences, Tsinghua University, Beijing 100084, China. ${ }^{4}$ Key Laboratory of Crop Germplasm Resources and Utilization, Ministry of Agriculture/The National Key Facility for Crop Gene Resources and Genetic Improvement/Institute of Crop Science, Chinese Academy of Agricultural Sciences, Beijing 100081, China. ${ }^{5}$ Crops Research Institute, Guangdong Academy of Agricultural Sciences, Guangdong 510000, China.

\section{Authors' contributions}

JZ carried out the peanut seed RNA isolation and sequence data analyses and drafted the manuscript. SL and JD designed the experiment and assisted in manuscript preparation. SC, JW, ZC, QZ, XL and YL prepared the plant materials and co-designed the experiments. All of the authors read and approved the final manuscript.

Received: 30 November 2011 Accepted: 12 March 2012 Published: 12 March 2012

\section{References}

1. Stephens AM, Dean LL, Davis JP, Osborne JA, Sanders TH: Peanuts, Peanut Oil, and Fat Free Peanut Flour Reduced Cardiovascular Disease Risk Factors and the Development of Atherosclerosis in Syrian Golden Hamsters. J Food Sci 2010, 75:H116-H122.

2. Kris-Etherton PM, Pearson TA, Wan Y, Hargrove RL, Moriarty K, Fishell V, Etherton TD: High-monounsaturated fatty acid diets lower both plasma cholesterol and triacylglycerol concentrations. Am J Clin Nutr 1999, 70:1009-1015. 
3. Kerckhoffs DA, Brouns F, Hornstra G, Mensink RP: Effects on the human serum lipoprotein profile of beta-glucan, soy protein and isoflavones, plant sterols and stanols, garlic and tocotrienols. J Nutr 2002, 132:2494-2505.

4. Griel AE, Eissenstat B, Juturu V, Hsieh G, Kris-Etherton PM: Improved diet quality with peanut consumption. J Am Coll Nutr 2004, 23:660-668

5. Ge SR, Sui QW, Yu SL: Simply analysis of the situation and countermeasures of peanut production in China. J Peanut Sci 1993, 4:3-6.

6. Ozcan MM: Some nutritional characteristics of kernel and oil of peanut (Arachis hypogaea L.). J Oleo Sci 2010, 59:1-5.

7. Fraser GE, Sabate J, Beeson WL, Strahan TM: A possible protective effect of nut consumption on risk of coronary heart disease. The Adventist Health Study. Arch Intern Med 1992, 152:1416-1424.

8. Bi YP, Liu W, Xia H, Su L, Zhao CZ, Wan SB, Wang XJ: EST sequencing and gene expression profiling of cultivated peanut (Arachis hypogaea L.). Genome 2010, 53:832-839.

9. Mortazavi A, Williams BA, McCue K, Schaeffer L, Wold B: Mapping and quantifying mammalian transcriptomes by RNA-Seq. Nature Methods 2008, 5:621-628.

10. Wang Z, Fang B, Chen J, Zhang X, Luo Z, Huang L, Chen X, Li Y: De novo assembly and characterization of root transcriptome using Illumina paired-end sequencing and development of cSSR markers in sweetpotato (Ipomoea batatas). BMC Genomics 2010, 11:726.

11. Wei W, Qi X, Wang L, Zhang Y, Hua W, Li D, LV H, Zhang X: Characterization of the sesame (Sesamum indicum L.) global transcriptome using Illumina paired-end sequencing and development of EST-SSR markers. BMC Genomics 2011, 12:451.

12. Lu T, Lu G, Fan D, Zhu C, Li W, Zhao Q, Feng Q, Zhao Y, Guo Y, Huang X, et al: Function annotation of the rice transcriptome at single-nucleotide resolution by RNA-seq. Genome Res 2010, 20:1238-1249.

13. Severin AJ, Woody JL, Bolon $Y-T$, Joseph B, Diers BW, Farmer AD, Muehlbauer GJ, Nelson RT, Grant D, Specht JE, et al: RNA-Seq Atlas of Glycine max: A guide to the soybean transcriptome. BMC Plant Biology 2010, 10:160.

14. Nagalakshmi U, Wang Z, Waern K, Shou C, Raha D, Gerstein M, Snyder M: The transcriptional landscape of the yeast genome defined by RNA sequencing. Science 2008, 320:1344-1349.

15. Wilhelm BT, Marguerat S, Watt S, Schubert F, Wood V, Goodhead I, Penkett CJ, Rogers J, Bahler J: Dynamic repertoire of a eukaryotic transcriptome surveyed at single-nucleotide resolution. Nature 2008, 453:1239-1243.

16. Cloonan N, Forrest AR, Kolle G, Gardiner BB, Faulkner GJ, Brown MK, Taylor DF, Steptoe AL, Wani S, Bethel G, et al: Stem cell transcriptome profiling via massive-scale mRNA sequencing. Nat Methods 2008, 5:613-619.

17. Zenoni S, Ferrarini A, Giacomelli E, Xumerle L, Fasoli M, Malerba G, Bellin D, Pezzotti M, Delledonne M: Characterization of Transcriptional Complexity during Berry Development in Vitis vinifera Using RNA-Seq. Plant Physiology 2010, 152:1787-1795.

18. Sultan M, Schulz MH, Richard $H$, Magen A, Klingenhoff A, Scherf $M$, Seifert M, Borodina T, Soldatov A, Parkhomchuk D, et al: A global view of gene activity and alternative splicing by deep sequencing of the human transcriptome. Science 2008, 321:956-960.

19. Filichkin SA, Priest HD, Givan SA, Shen R, Bryant DW, Fox SE, Wong WK, Mockler TC: Genome-wide mapping of alternative splicing in Arabidopsis thaliana. Genome Res 2010, 20:45-58.

20. Marioni JC, Mason CE, Mane SM, Stephens M, Gilad Y: RNA-seq: An assessment of technical reproducibility and comparison with gene expression arrays. Genome Res 2008, 18:1509-1517.

21. Fullwood MJ, Wei CL, Liu ET, Ruan Y: Next-generation DNA sequencing of paired-end tags (PET) for transcriptome and genome analyses. Genome Res 2009, 19:521-532.

22. Torres TT, Metta M, Ottenwalder B, Schlotterer C: Gene expression profiling by massively parallel sequencing. Genome Res 2008, 18:172-177.

23. Hegedus Z, Zakrzewska A, Agoston VC, Ordas A, Racz P, Mink M, Spaink HP, Meijer $A H$ : Deep sequencing of the zebrafish transcriptome response to mycobacterium infection. Mol Immunol 2009, 46:2918-2930.

24. Clark MS, Thorne MA, Vieira FA, Cardoso JC, Power DM, Peck LS: Insights into shell deposition in the Antarctic bivalve Laternula elliptica: gene discovery in the mantle transcriptome using 454 pyrosequencing. BMC Genomics 2010, 11:362.
25. Iorizzo M, Senalik DA, Grzebelus D, Bowman M, Cavagnaro PF, Matvienko M, Ashrafi $H$, Van Deynze A, Simon PW: De novo assembly and characterization of the carrot transcriptome reveals novel genes, new markers, and genetic diversity. BMC Genomics 2011, 12:389.

26. Graham IA, Besser K, Blumer S, Branigan CA, Czechowski T, Elias L, Guterman I, Harvey D, Isaac PG, Khan AM, et al: The genetic map of Artemisia annua L. identifies loci affecting yield of the antimalarial drug artemisinin. Science 2010, 327:328-331.

27. Oliver RE, Lazo GR, Lutz JD, Rubenfield MJ, Tinker NA, Anderson JM, Wisniewski Morehead NH, Adhikary D, Jellen EN, Maughan PJ, et al: Model SNP development for complex genomes based on hexaploid oat using high-throughput 454 sequencing technology. BMC Genomics 2011, 12:77.

28. Chen SL, Li YR, Xu GZ, Cheng ZS: Simulation on Oil Accumulation Characteristics in Different High-Oil Peanut Varieties. Acta Agronomica Sinica 2008, 34:142-149.

29. Li R, Zhu H, Ruan J, Qian W, Fang X, Shi Z, Li Y, Li S, Shan G, Kristiansen K, et al: De novo assembly of human genomes with massively parallel short read sequencing. Genome Res 2010, 20:265-272.

30. Altschul SF, Madden TL, Schaffer AA, Zhang J, Zhang Z, Miller W, Lipman DJ: Gapped BLAST and PSI-BLAST: a new generation of protein database search programs. Nucleic Acids Res 1997, 25:3389-3402.

31. Conesa A, Gotz S, Garcia-Gomez JM, Terol J, Talon M, Robles M: Blast2GO: a universal tool for annotation, visualization and analysis in functional genomics research. Bioinformatics 2005, 21:3674-3676.

32. Ye J, Fang L, Zheng H, Zhang Y, Chen J, Zhang Z, Wang J, Li S, Li R, Bolund L: WEGO: a web tool for plotting GO annotations. Nucleic Acids Res 2006, 34:W293-W297.

33. Kanehisa M, Araki M, Goto S, Hattori M, Hirakawa M, Itoh M, Katayama T, Kawashima S, Okuda S, Tokimatsu T, et al: KEGG for linking genomes to life and the environment. Nucleic Acids Res 2008, 36:D480-D484.

34. Liang X, Chen X, Hong Y, Liu H, Zhou G, Li S, Guo B: Utility of EST-derived SSR in cultivated peanut (Arachis hypogaea L.) and Arachis wild species. BMC Plant Biology 2009, 9:35.

doi:10.1186/1471-2164-13-90

Cite this article as: Zhang et al:: De novo assembly and Characterisation of the Transcriptome during seed development, and generation of genic-SSR markers in Peanut (Arachis hypogaea L.). BMC Genomics 2012 13:90.

\section{Submit your next manuscript to BioMed Central and take full advantage of:}

- Convenient online submission

- Thorough peer review

- No space constraints or color figure charges

- Immediate publication on acceptance

- Inclusion in PubMed, CAS, Scopus and Google Scholar

- Research which is freely available for redistribution

Submit your manuscript at www.biomedcentral.com/submit
C Biomed Central 